\title{
VARIABILIDADE DA ÁGUA DISPONIVEL DE UMA TERRA ROXA ESTRUTURADA LATOSSÓLICA ${ }^{1}$
}

\author{
S.O.MORAES ${ }^{2}$; P.L.LIBARDI ${ }^{2}$ \\ Departamento de Fisica e Meteorologia, ESALQ/USP, C.P. 9 - CEP: 13418-900-Piracicaba,SP.
}

RESUMO: A partir de 250 curvas de retenção da água no solo, elaboradas com amostras indeformadas coletadas de uma área de $6250 \mathrm{~m}^{2}$ de uma Terra Roxa Estruturada Latoss6lica de Piracicaba,SP, foram calculados quatro conjuntos de valores de água disponível assumindo-se $-1 \times 10^{3},-6 \times 10^{3},-1 \times 10^{4}$ e $-3 \times 10^{4} \mathrm{~Pa}$ como possíveis valores de potencial mátrico correspondentes á capacidade de campo e -1,5x10 Pa um possivel valor correspondente ao ponto de murchamento permanente. Foram feitas medidas de posição (média), variabilidade (coeficiente de variação, assimetria e curtose) e número necessário de amostras para estimar a média a um dado nível de probabilidade a fím de quantificar a variabilidade e a sensibilidade dos resultados em cada conjunto e entre conjuntos de valores de água disponível. A análise dos resultados mostrou que a variabilidade da água disponível, obtida à partir de dois valores de umidade da Curva de Retenção é muito maior que a variabilidade de cada valor individualmente. Ou seja, embora as variáveis envolvidas possam ser as mesmas, o grau de variabilidade (expresso, por exemplo, pelo coeficiente de variação) ou a sensibilidade das medidas (expressa pelo número necessário de amostras para estimar a média dentro de um determinado intervalo de confiança) pode ser bem distinto, indicando que nem sempre resultados de uma amostragem realizada com determinado objetivo podera servir a outros, embora possam tratar-se de váriaveis dependentes.

Deacritores: curva de retenção da água no solo, variabilidade, água disponível, terra roxa estruturada latossólica.

\section{AVAILABLE SOIL-WATER VARIABILITY OF A "TERRA ROXA ESTRUTURADA LATOSSOLICA" (RHODIC KANHAPLUDALF)}

SUMMARY: From 250 soil-water retention curves of an area of $6250 \mathrm{~m}^{2}$ of a "Terra Roxa Estruturada Latoss6lica" (Rhodic Kanhapludaln) located in Piracicaba,SP, four sets of available soil-water were calculated assuming field capacity values based on soil-water contents corresponding to $-1 \times 10^{3},-6 \times 10^{3},-1 \times 10^{4}$ and $-3 \times 10^{4} P a$ of soil water matric potential; and permanent wilting point based on soil-water contents corresponding to $-1,5 \times 10^{\circ}$ Pa. Aiming to quantify the variability and the sensibility of the results for each set and among sets of soil available water values, the following calculations were made: position measurement (mean), variability (coefficient of variation, assimetry and kurtosis) and the necessary number of samples to estimate the mean at a specific probability level. The analysis of the results has shown that the variability of available soil-water values is much greater than the variability of field capacity and of permanent wilting point values used in the calculation. That is, even though the envolved variables can be the same, the degree of variability (expressed by the necessary number of samples needed to estimate the mean within a choosen interval confidence) can be very distinct, indicating that the results of a sampling, carried out for one specific objective can not always be used for another objective, even being dependent variables.

Key words: soil-water retention curve, variability, soil available water, Rhodic Kanhapludalf.

\section{INTRODUÇAO}

Os conceitos de Capacidade de Campo, de ponto de murchamento permanente $e$, consequentemente, de água disponível, têm sido alvo de constante discussão na literatura pertinente
(REICHARDT, 1988). Basicamente as principais dificuldades envolvidas são: 1) Grande variação nos valores de potencial mátrico que correspondem à capacidade de campo e ao ponto de murchamento permanente. Apesar disso há grande tendência em assumirem-se valores únicos independentemente do

\footnotetext{
1 Parte da Tese de Doutorado do primeiro Autor, junto ao Curso de Pós-Graduação em Solos e Nutrição de Plantas da ESALQ/USP.

${ }^{2}$ Bolsista do CNPq.
}

Sci. agric., Piracicaba, 50(3):404-412, out./dez., 1993 
tipo de solo, por exemplo, $6 \times 10^{3} \mathrm{~Pa}$ ou $3 \times 10^{4} \mathrm{~Pa}$

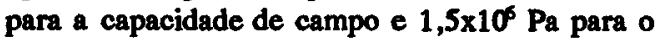
ponto de murchamento permanente (LEAMER \& SHAW, 1941; RICHARDS, 1949; COLMAN, 1917; JAMISON \& KROTH, 1985; SOUZA, 1989); 2) Correspondência entre valores medidos em laboratório e no campo (SALTER \& HAWORTH, 1961; BORGES \& MEDINA, 1981; STEVENSON, 1982; RATLIFF, RITCHIE \& CASSEL, 1983); 3) verificação das condições sob as quais os conceitos foram definidos, particularmente do fim da drenagem livre para medida da Capacidade de Campo (CASSEL \& NIELSEN, 1986; SOUZA, 1989).

Apesar dessas dificuldades, a água disponível $\epsilon$ amplamente utilizada em estudos da água no solo e rotineiramente os pontos da curva de retenção da água no solo (potencial mátrico é a variável independente $e$ umidade à base de massa ou de volume é a variável dependente) são obtidos em condições de laboratório. $O$ presente trabalho utiliza, a partir de 250 curvas de retenção da água no solo obtidas por MORAES (1991) alguns valores de potencial mátrico aceitos na literatura para o cálculo da água disponível: $1 \times 10^{3} ; 6 \times 10^{3}$; $1 \times 10^{4}$ e $3 \times 10^{4} \mathrm{~Pa}$ para a Capacidade de Campo e $1,5 \times 10^{\circ}$ para $O$ ponto de murchamento permanente(LEAMER \& SHAW, 1941; RICHARDS, 1949; COLMAN, 1917; JAMISON \& KROTH, 1985; SOUZA, 1989). O objetivo do trabalho e verificar como fica a variabilidade dos vários conjuntos de valores de água disponível obtidos em comparação com a variabilidade dos valores da capacidade de campo e do ponto de murchamento permanente que lhe deram origem (SILVA,1988) enfocando os seguintes objetivos especificos: a) verificar a normalidade da distribuição para os vários conjuntos de valores de agua disponível; b) quantificar a variabilidade e a sensibilidade das medidas em cada conjunto e entre conjuntos de valores de água disponível.

\section{MATERIAL E MÉTODOS}

Foram coletadas 250 amostras com estrutura indeformada de uma Terra Roxa Estruturada Latossólica situada próxima ao Posto Agrometeorológico do Departamento de Física e Meteorologia da ESALQ/USP. A profundidade de coleta foi $25 \mathrm{~cm}$ (MORAES, 1991), segundo uma amostragem sistemática (WEBSTER, 1977; PETERSEN \& CALVIN, 1986), obedecendo a um arranjo com espaçamento regular de $5 \mathrm{~m}$ entre os pontos amostrados, resultando numa malha quadriculada de 25 linhas e 10 colunas.

Com as amostras, foram obtidas 250 Curvas de Retenção da Água no Solo, conforme MORAES (1991) e MORAES et al. (1991 a e b). Cada curva constou de 8 pontos $(\psi \mathrm{m} \times \mathrm{U})$ sendo $\psi_{\mathrm{m}_{1}}=5 \times 10^{2} ; \psi_{\mathrm{m} 2}=1 \times 10^{3} ; \psi_{\mathrm{m} 3}=6,0 \times 10^{3} ;$ $\psi_{\mathrm{m} 4}=1 \times 10^{4} ; \psi_{\mathrm{ms}}=3 \times 10^{4} ; \psi_{\mathrm{mb}}=8 \times 10^{4} ; \psi_{\mathrm{m} 7}$ $=3 \times 10^{5}$ e $\psi_{\mathrm{m} 8}=1,5 \times 10^{6} \mathrm{~Pa}$ e $\mathrm{U}(\mathrm{g} / \mathrm{g})$ as correspondentes umidades a base de massa. $A$ partir desses dados, calcularam-se os possíveis valores de água disponível, pela diferença entre as umidades a Capacidade de Campo e o ponto de murchamento permanente, sendo o resultado expresso à base de massa para não ser influenciado pela variabilidade da densidade do solo. A escolha dos possíveis valores de tensão correspondentes à capacidade de campo e ao ponto de murchamento permanente baseou-se na literatura, adotando-se todos os valores que foram encontrados, uma vez que a intenção era questionar a variabilidade e não - conceito ou sua utilização. Assim utilizou-se os seguintes valores de tensão correspondentes à Capacidade de Campo: 1) $1 \times 10^{3} \mathrm{~Pa}$ (JAMISON \&

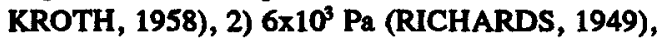
3) $1 \times 10^{4} \mathrm{~Pa}$ (JAMISON \& KROTH, 1986 RICHARDS, 1934) e 4) $3 \times 10^{4} \mathrm{~Pa}$ (COLMAN, 1947) e para o ponto de murchamento permanente $1,5 \times 10^{6} \mathrm{~Pa}$ (SALTER \& HAWORTH, 1961), obtendo-se então quatro conjuntos de valores de água disponível. Para cada conjunto foram analisados os seguintes parâmetros estatísticos:

a) medida de posição: média aritmética $\left(x_{m}\right)$, (CLARCK \& HOSKING 1986); b) medidas de dispersão (ou variabilidade): coeficiente de variação (cv $=100 . s / x_{m}$ onde s é o desvio padrão e $x_{m}$ a média aritmética, (CLARCK \& HOSKING, 1986), assimetria $\left(b_{1}=m^{\prime}{ }_{3} / m_{2}^{\prime}\right)$ e curtose $\left(b_{2}=\right.$ $m_{4}^{\prime} / m_{2}^{\prime}$ ) onde $m_{2}^{\prime}, m_{3}^{\prime}$ e $m_{4}^{\prime}$ são respectivamente - segundo, terceiro e quarto momentos centrados na média, (LANDIM, 1985). Para a distribuição normal, a assimetria apresenta valores em tomo de zero e a curtose em torno de três (CLARCK \& HOSKING, 1986); c) número necessário de amostras para estimar a média a um dado nível de probabilidade (CLINE, 1944; ALJIBURY \& EVANS, 1961; WARRICK \& NIELSEN, 1980), isto $\varepsilon$, se uma estimativa da variância $\epsilon$ disponivel (por amostragens prévias ou conhecimento prévio da população) então uma estimativa do número de amostras necessárias em amostragens futuras, para uma dada precisão com um limite especificado de probabilidade pode ser obtido da seguinte equação: 
$\mathrm{N}=\left(\mathrm{t}_{\alpha}\right)^{2} \times \mathrm{s}^{2} / \mathrm{D}^{2}$, onde $\mathrm{t}_{\alpha}$ é o $t$ de Student com n-1 graus de liberdade, ao nível $\alpha$ de probabilidade, $D$ é o desvio permitido e s é o desvio padrão (PETERSEN \& CALVIN, 1986); d) medida por razão (para determinados tipos de variáveis, a posição de um particular resultado da variável pode ser obtido expressando-se este resultado como uma razão entre algum outro resultado ou valor. No caso optou-se pelo resultado padronizado, também denominado variável reduzida $Z$, definida da seguinte forma: $\mathrm{Zi}=\left(\mathrm{x}_{1}-\mathrm{x}_{m}\right) / \mathrm{s}$, para qualquer valor $X_{i}$ assumido por uma variável, onde $x_{1}$ é o i-ésimo valor da variável, $x_{m}$ é a média aritmética e $s$ o desvio padrão CLARCK \& HOSKING, 1986).

No presente caso a escolha dessa forma para expressar os diversos conjuntos de valores de água disponível deve-se a que esta representação, proporcionando média nula e sendo expressa em unidades de desvio padrão, faz com que sejam comparáveis os vários conjuntos de água disponível, mesmo que tenham médias distintas, como é o caso, e deste ponto de vista apresenta a mesma utilidade que o coeficiente de variação.

As medidas de posição, dispersão $e$ as distribuições de freqüencia em termos de variáveis reduzidas foram elaboradas utilizando-se o pacote STATGRAPHICS (1987). O número necessário de amostras para estimar a média foi obtido à partir do resultado de variância fornecido pelo STATGRAPHICS (1987) mais as TABELAS estatísticas para valores de $t$ aos níveis especificados de significância, desvios permitidos e (n-1) graus de liberdade.

\section{RESULTADOS E DISCUSSÃO}

A TABELA 1, obtida à partir dos dados de umidade à base de massa apresentados em MORAES (1991) e MORAES et al. (1992 a,b) apresenta alguns parâmetros estatísticos para análise de medidas de posição e dispersão, assumindo-se vários valores de umidade à Capacidade de Campo (CC) correspondentes à vários valores de tensão aceitos pelos pesquisadores (JAMISON \& KROTH, 1985; RICHARDS, 1949; COLMAN, 1947) para vários tamanhos de amostra $(250,143$ e 116 elementos), segundo critérios discutidos em MORAES (1991) e MORAES et al. $(1991$, a).

Inicialmente, comparando-se visualmente as médias obtidas observa-se um sensível decréscimo na água disponível (AD) expressa em porcentagem (AD\%) à medida que se aumenta as tensões assumidas para $\mathrm{CC} \mathrm{e}$ um ligeiro aumento à medida que se compara para a mesma tensão, com valores decrescentes de número de amostras $(N)$.

A primeira observação $E$ trivial e a outra pode ser entendida à partir de MORAES et al. (1991, b) onde se observa que o descarte de amostras-problema provocou maior variação (no caso diminuição) nas umidades correspondentes ao ponto de murchamento permanente (PMP) e não nas demais assumidas para a CC, portanto, a subtração de valores consecutivamente menores de valores praticamente constantes levou a aumentos consecutivos na $\mathrm{AD} \%$, para uma mesma tensão.

Uma análise dos coeficientes de assimetria e curtose apresentados, à partir dos parâmetros de GOES (1980), para os vários tamanhos de amostra considerados mostra que:

$\left.1^{\circ}\right)$ para $N=250$ :

AD\% $\left(C C=1 \times 10^{3} \mathrm{~Pa}\right)$ apresenta assimetria exagerada e a curva é leptocúrtica. AD\% (CC = $6 \times 10^{3} \mathrm{~Pa}$ ) apresenta simetria admissivel e a curva é leptocúrtica. AD\% (CC $\left.=1 \times 10^{4} \mathrm{~Pa}\right)$ apresenta assimetria moderada e a curva é leptocúrtica. AD\% $\left(C C=3 \times 10^{4} \mathrm{~Pa}\right)$ apresenta simetria admissível e a curva é leptocúrtica.

$\left.2^{\circ}\right)$ para $N=143$ :

AD\% $\left(C C=1 \times 10^{3} \mathrm{~Pa}\right)$ apresenta simetria admissivel e a curva é leptocúrtica. AD\% (CC = $6 \times 10^{3} \mathrm{~Pa}$ ) apresenta simetria admissivel e a curva é leptocúrtica. AD\% $\left(C C=1 \times 10^{6} \mathrm{~Pa}\right)$ apresenta assimetria moderada e a curva é leptocúrtica. AD\% $\left(C C=3 \times 10^{\circ} \mathrm{Pa}\right)$ apresenta assimetria exagerada e a curva é leptocúrtica.

$\left.3^{\circ}\right)$ para $N=116$ :

AD\% $\left(C C=1 \times 10^{3} \mathrm{~Pa}\right)$ apresenta simetria admissivel e a curva é platicúrtica. AD\% (CC $=6$ $x 10^{3} \mathrm{~Pa}$ ) apresenta assimetria moderada e a curva É leptocúrtica. $\mathbf{A D \%}\left(\mathrm{CC}=1 \times 10^{4} \mathrm{~Pa}\right)$ apresenta assimetria moderada e a curva é leptocúrtica. AD\% $\left(C C=3 \times 10^{4} \mathrm{~Pa}\right)$ apresenta simetria admissivel e a curva é platicúrtica. Ou seja, aplicando-se o método dos momentos de assimetria e curtose para reconhecimento da distribuição normal (LANDAM, 1985; HOFFMANN, 1980), os valores de agua disponível que mais se encaixariam seriam:

$A D \%\left(C C=6 \times 10^{3} \mathrm{~Pa}\right)$ para $N=250$; $A D \%\left(C C=1 \times 10^{3}\right.$ e $\left.6 \times 10^{3} \mathrm{~Pa}\right)$ para $N=143$; $A D \%\left(C C=1 \times 10^{3}\right.$ e $\left.3 \times 10^{4} \mathrm{~Pa}\right)$ para $N=116$. 
TABELA 1. Média, assimetria, curtose e coeficiente de variação (CV\%)da água disponível (AD\%), calculada para o ponto de murchamento permanente (PMP) correspondente a $1,5 \times 10^{0} \mathrm{~Pa}$ e vários valores possíveis de Capacidade de Campo (CC) $\left(1 \times 10^{3} ; 6 \times 10^{3} ; 1 \times 10^{4}\right.$ e $\left.3 \times 10^{4} \mathrm{~Pa}\right)$ para 250,143 e 116 amostras.

\begin{tabular}{|c|c|c|c|c|c|c|c|c|c|c|c|c|}
\hline \multirow[b]{2}{*}{$\mathrm{AD}(\%)^{(1)}$} & \multicolumn{3}{|c|}{ Médias } & \multicolumn{3}{|c|}{ Assimetria } & \multicolumn{3}{|c|}{ Curtose } & \multicolumn{3}{|c|}{ CV\% } \\
\hline & $\begin{array}{l}\mathrm{N}= \\
250 \\
\end{array}$ & $\begin{array}{l}N= \\
143\end{array}$ & $\begin{array}{l}\mathrm{N}= \\
116\end{array}$ & $\begin{array}{l}\mathrm{N}= \\
250\end{array}$ & $\begin{array}{l}N= \\
143\end{array}$ & $\begin{array}{l}N= \\
116\end{array}$ & $\begin{array}{l}\mathrm{N}= \\
250\end{array}$ & $\begin{array}{l}N= \\
143\end{array}$ & $\begin{array}{l}N= \\
116\end{array}$ & $\begin{array}{l}\mathrm{N}= \\
250\end{array}$ & $\begin{array}{l}\mathrm{N}= \\
143\end{array}$ & $\begin{array}{l}\mathrm{N}= \\
116\end{array}$ \\
\hline $\mathrm{AD}(\%)^{(2)}$ & 11 & 12 & 16 & 1,57 & 0,05 & 0,32 & 10,77 & 0,37 & $-0,04$ & 24 & 19 & 17 \\
\hline $\mathrm{AD}(\%)^{(i)}$ & 8 & 9 & 9 & 0,48 & 0,37 & 0,52 & 0,26 & 0,05 & 0,04 & 23 & 20 & 18 \\
\hline $\mathrm{AD}(\%)^{(4)}$ & 7 & 8 & 8 & 0,55 & 0,67 & 0,68 & 0,56 & 0,33 & 0,27 & 24 & 21 & 21 \\
\hline $\mathrm{AD}(\%)^{(5)}$ & 5 & 6 & 7 & $-0,24$ & $-1,39$ & 0,26 & 3,78 & 8,75 & $-0,68$ & 36 & 32 & 24 \\
\hline
\end{tabular}

(1) $\mathrm{AD}(\%)=(\mathrm{CC}-\mathrm{PMP}) .100$, (2) $\mathrm{CC}=1 \times 10^{3} \mathrm{~Pa},(3) \mathrm{CC}=6 \times 10^{3} \mathrm{~Pa},(4) \mathrm{CC}=1 \times 10^{4} \mathrm{~Pa},(5) \mathrm{CC}=3 \times 10^{4} \mathrm{~Pa}$.

TABELA 2. Frequências de água disponível (AD\%) expressas em unidades de desvio padrão ( $Z$ i) calculada para o ponto de murchamento permanente (PMP) correspondente a $1,5 \times 10^{6} \mathrm{~Pa}$ e vários valores possíveis de Capacidade de Campo (CC) $\left(1 \times 10^{3} ; 6 \times 10^{3} ; 1 \times 10^{4}\right.$ e $\left.3 \times 10^{4} \mathrm{~Pa}\right)$, para 250 amostras.

\begin{tabular}{ccccccc}
\hline \hline & \multirow{2}{*}{ Limite } & Limite & \multicolumn{4}{c}{ Frequências $(1)$} \\
\cline { 4 - 7 } Classes & Inferior & Superior & $\begin{array}{c}\mathrm{CC}=1 \times 10^{3} \\
\mathrm{~Pa}\end{array}$ & $\begin{array}{c}\mathrm{CC}=6 \times 10^{3} \\
\mathrm{~Pa}\end{array}$ & $\begin{array}{c}\mathrm{CC}=1 \times 10^{4} \\
\mathrm{~Pa}\end{array}$ & $\begin{array}{c}\mathrm{CC}=3 \times 10^{4} \\
\mathrm{~Pa}\end{array}$ \\
\hline 1 & $-6,500$ & $-5,647$ & 0 & 0 & 0 & 1 \\
2 & $-5,647$ & $-4,794$ & 0 & 0 & 0 & 0 \\
3 & $-4,794$ & $-3,941$ & 0 & 0 & 0 & 0 \\
4 & $-3,941$ & $-3,088$ & 0 & 0 & 0 & 0 \\
5 & $-3,088$ & $-2,235$ & 2 & 1 & 0 & 0 \\
6 & $-2,335$ & $-1,382$ & 15 & 15 & 19 & 8 \\
7 & $-1,382$ & 0,529 & 52 & 65 & 56 & 69 \\
8 & $-0,529$ & 0,324 & 95 & 81 & 89 & 97 \\
9 & $-0,324$ & 1,176 & 62 & 58 & 61 & 35 \\
10 & 1,176 & 2,029 & 20 & 22 & 16 & 33 \\
11 & 2,029 & 2,882 & 3 & 7 & 5 & 7 \\
12 & 2,882 & 3,735 & 0 & 1 & 4 & 0 \\
13 & 3,735 & 4,588 & 0 & 0 & 0 & 0 \\
14 & 4,588 & 5,441 & 0 & 0 & 0 & 0 \\
15 & 5,441 & 6,294 & 0 & 0 & 0 & 0 \\
16 & 6,294 & 7,147 & 0 & 0 & 0 & 0 \\
17 & 7,147 & 8,000 & 1 & 0 & 0 & 0 \\
\hline \hline
\end{tabular}

(1) Freqüências da variável reduzida $\mathrm{Zi}=\left(\mathrm{AD} \%-\mathrm{AD} \%_{\mathrm{m}}\right) / \mathrm{S}$, onde $\mathrm{AD} \%_{\mathrm{m}}$ é a média aritmética dos valores de AD\%. 
TABELA 3. Frequências de água disponível (AD\%) expressas em unidades de desvio padrão ( $\mathrm{Zi}$ ) calculada para o ponto de murchamento permanente (PMP) correspondente a $1,5 \times 10^{\circ} \mathrm{Pa}$ e vários valores possíveis de Capacidade de Campo (CC) $\left(1 \times 10^{3} ; 6 \times 10^{3} ; 1 \times 10^{4}\right.$ e $\left.3 \times 10^{4} \mathrm{~Pa}\right)$, para 143 amostras.

\begin{tabular}{|c|c|c|c|c|c|c|}
\hline \multirow[b]{2}{*}{ Classes } & \multirow{2}{*}{$\begin{array}{l}\text { Limite } \\
\text { Inferior }\end{array}$} & \multirow{2}{*}{$\begin{array}{c}\text { Limite } \\
\text { Superior }\end{array}$} & \multicolumn{4}{|c|}{ Frequências ${ }^{(1)}$} \\
\hline & & & $\begin{array}{c}\mathrm{CC}=1 \times 10^{3} \\
\mathrm{~Pa}\end{array}$ & $\begin{array}{c}\mathrm{CC}=6 \times 10^{3} \\
\mathrm{~Pa}\end{array}$ & $\begin{array}{c}\mathrm{CC}=1 \times 10^{4} \\
\mathrm{~Pa}\end{array}$ & $\begin{array}{c}\mathrm{CC}=3 \times 10^{4} \\
\mathrm{~Pa}\end{array}$ \\
\hline 1 & $-6,500$ & $-5,647$ & 0 & 0 & 0 & 1 \\
\hline 2 & $-5,647$ & $-4,794$ & $\mathbf{0}$ & 0 & 0 & $\mathbf{0}$ \\
\hline 3 & $-4,794$ & $-3,941$ & 0 & 0 & 0 & 0 \\
\hline 4 & $-3,941$ & $-3,088$ & 1 & 0 & 0 & $\mathbf{0}$ \\
\hline 5 & $-3,088$ & $-2,235$ & 0 & 1 & 0 & $\mathbf{0}$ \\
\hline 6 & $-2,335$ & $-1,382$ & 7 & 11 & 7 & 5 \\
\hline 7 & $-1,382$ & 0,529 & 38 & 35 & 42 & 39 \\
\hline 8 & $-0,529$ & 0,324 & 53 & 42 & 40 & 44 \\
\hline 9 & $-0,324$ & 1,176 & 23 & 38 & 38 & 40 \\
\hline 10 & 1,176 & 2,029 & 18 & 9 & 8 & 12 \\
\hline 11 & 2,029 & 2,882 & 3 & 7 & 8 & 2 \\
\hline 12 & 2,882 & 3,735 & 0 & 0 & 0 & 0 \\
\hline 13 & 3,735 & 4,588 & 0 & 0 & 0 & 0 \\
\hline 14 & 4,588 & 5,441 & 0 & 0 & 0 & 0 \\
\hline 15 & 5,441 & 6,294 & 0 & 0 & 0 & 0 \\
\hline 16 & 6,294 & 7,147 & 0 & 0 & $\mathbf{0}$ & 0 \\
\hline 17 & 7,147 & 8,000 & 0 & 0 & 0 & 0 \\
\hline
\end{tabular}

(1) Freqüencias da variável reduzida $\mathrm{Zi}=\left(\mathrm{AD} \%-\mathrm{AD} \%_{\mathrm{m}}\right) / \mathrm{s}$, onde $\mathrm{AD} \%_{\mathrm{m}}$ é a média aritmética dos valores de $\mathrm{AD} \%$.

por apresentarem simetria admissivel e baixos valores dos coeficientes de curtose.

A importância da presente discussão não se refere apenas à relevância do conhecimento do tipo de distribuição, já discutida em MORAES et al. $(1991$, b), mas as modificações no tipo de distribuição, quando se compõem variáveis para obtenção de uma outra.
Assim, as distribuições empíricas de frequência para valores de umidade à base de massa com $N=143$ e $N=116$ apresentaram-se bastante próximas à normal (MORAES, 1991 b), mas quando se compoem estes valores para obterse água disponível, esta não se comportou da mesma forma, isto é, a normalidade foi perdida. Do ponto de vista prático, a colocação acima indica que nem sempre se poderá lançar mão de uma 
TABELA 4. Frequências de água disponível (AD\%) expressas em unidades de desvio padrão (Zi) calculada

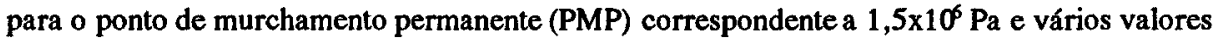
possíveis de Capacidade de Campo (CC) $\left(1 \times 10^{3} ; 6 \times 10^{3} ; 1 \times 10^{4}\right.$ e $\left.3 \times 10^{4} \mathrm{~Pa}\right)$, para 116 amostras.

\begin{tabular}{|c|c|c|c|c|c|c|}
\hline \multirow[b]{2}{*}{ Classes } & \multirow{2}{*}{$\begin{array}{l}\text { Limite } \\
\text { Inferior }\end{array}$} & \multirow{2}{*}{$\begin{array}{l}\text { Limite } \\
\text { Superior }\end{array}$} & \multicolumn{4}{|c|}{ Frequências ${ }^{(1)}$} \\
\hline & & & $\begin{array}{c}\mathrm{CC}=1 \times 10^{3} \\
\mathrm{~Pa}\end{array}$ & $\begin{array}{c}\mathrm{CC}=6 \times 10^{3} \\
\mathrm{~Pa}\end{array}$ & $\begin{array}{c}\mathrm{CC}=1 \times 10^{4} \\
\mathrm{~Pa}\end{array}$ & $\begin{array}{c}\mathrm{CC}=3 \times 10^{4} \\
\mathrm{~Pa}\end{array}$ \\
\hline 1 & $-6,500$ & $-5,647$ & 0 & 0 & $\mathbf{0}$ & 1 \\
\hline 2 & $-5,647$ & $-4,794$ & 0 & 0 & $\mathbf{0}$ & 0 \\
\hline 3 & $-4,794$ & $-3,941$ & 0 & 0 & 0 & 0 \\
\hline 4 & $-3,941$ & $-3,088$ & 0 & 0 & 0 & 0 \\
\hline 5 & $-3,088$ & $-2,235$ & 1 & 0 & 0 & 0 \\
\hline 6 & $-2,335$ & $-1,382$ & 6 & 7 & 5 & 7 \\
\hline 7 & $-1,382$ & 0,529 & 28 & 33 & 36 & 34 \\
\hline 8 & $-0,529$ & 0,324 & 45 & 35 & 35 & 28 \\
\hline 9 & $-0,324$ & 1,176 & 18 & 26 & 26 & 33 \\
\hline 10 & 1,176 & 2,029 & 15 & 9 & 8 & 10 \\
\hline 11 & 2,029 & 2,882 & 3 & 6 & 6 & 4 \\
\hline 12 & 2,882 & 3,735 & 0 & 0 & 0 & $\mathbf{0}$ \\
\hline 13 & 3,735 & 4,588 & 0 & 0 & 0 & 0 \\
\hline 14 & 4,588 & 5,441 & 0 & 0 & 0 & 0 \\
\hline 15 & 5,441 & 6,294 & $\mathbf{0}$ & 0 & 0 & $\mathbf{0}$ \\
\hline 16 & 6,294 & 7,147 & 0 & 0 & 0 & 0 \\
\hline 17 & 7,147 & 8,000 & 0 & 0 & 0 & 0 \\
\hline
\end{tabular}

(1) Freqüencias da variável reduzida $\mathrm{Zi}=\left(\mathrm{AD} \%-\mathrm{AD} \%_{\mathrm{m}}\right) / \mathrm{s}$, onde $\mathrm{AD} \%_{\mathrm{m}}$ é a média aritmética dos valores de $\mathrm{AD} \%$.

caracterização hídrica de um solo, determinada por exemplo, à partir de três repetições e utilizá-la para obtenção de uma outra variável, no caso, água disponível e obter-se o mesmo grau de variabilidade. De fato, o afastamento da normal já sugere que provavelmente $o$ coeficiente de variação já será maior para $\mathrm{AD} \%$ do que para as umidades individuais e que provavelmente haverá necessidade de maior número de amostras para estimar a média, a fím de que o Teorema do Limite Central se aplique (HOFFMAN, 1980; WARRICK \& NIELSEN, 1980).

A observação da TABELA 1 confirma o aumento dos coeficientes de variação para os três tamanhos de amostra considerados, quando comparados aos das umidades individualmente (MORAES et al. 1991, b). No caso, passaram de baixa (cv < $12 \%)$ para média variação $(12 \leq \mathrm{cv}$ $\leq 52 \%$ ), segundo os critérios de WARRICK \& NIELSEN (1980). Resultados análogos também 
foram obtidos por SILVA (1988) que utilizou as tensões de 0,01 e 0,033 MPa para obtenção das umidades correspondentes à $\mathrm{CC}$.

A utilização do indice de variação em substituição ao coeficiente de variação, conforme proposto por GOMES (1991) também mostraria o aumento obtido para o coeficiente de variação, embora, como colocado pelo autor este índice também fosse aumentando quando se diminui o tamanho da amostra. Não invalida no entanto, a comparação dos coeficientes de variação para mesmos tamanhos de amostra e propriedades distintas, como no caso, a comparação entre água disponível e os dados de umidade à base de massa (MORAES et al. 1991, b) que thes deram origem.

Outro fato que chama a atenção na TABELA 1, é o aumento relativo do coeficiente de variação para $A D \%$ com a $C C$ correspondendo a 3 $\times 10^{4} \mathrm{~Pa}$, particularmente para valores de $\mathrm{N}=$ 250 e $\mathrm{N}=143$. Analogamente ao efetuado em MORAES et al. (1991, b), as TABELAS 2, 3 e 4, que expressam as frequências de valores de água disponível em unidades de desvio padrão indicam os valores discrepantes (classe 1 de frequência), responsáveis pela distorção, explicando ainda os sinais encontrados na TABELA 1 para o momento de assimetria.

Embora já se tenha discutido anteriormente, quais valores de $\mathrm{AD} \%$ seriam mais adequados para utilização da equação que permite o cálculo do número de amostras necessário para estimar-se a média a um dado nivel de probabilidade (CLINE, 1944; ALJIBURY \& EVANS, 1961; WARRICK \& NIELSEN, 1980; MORAES et al., 1991 b), a aplicação indistinta para os vários valores de $\mathrm{AD \%}$, para os vários tamanhos de amostra considerados, forneceria os valores apresentados na TABELA 5.

Os altos valores observados em alguns casos (AD\% para CC $=1 \times 10^{3} \mathrm{~Pa}$ e CC $=3 \times$ $10^{4} \mathrm{~Pa}$ para $\mathrm{N}=250$ ou para $\mathrm{CC}=3 \times 10^{4} \mathrm{~Pa}$ para $N=143$ ) podem ser explicados pelas TABELAS $1,2,3$ e 4, na primeira pelos coeficientes de assimetria e curtose e nas demais pela presença de valores discrepantes, de qualquer forma, pelo afastamento da normal, confirmando assim a observação feita anteriormente, quanto ao aumento do número de amostras, quando da composição de duas variáveis para obtenção de uma terceira.
Ressalte-se que a TABELA 5 foi elaborada para vários tamanhos de amostra, conforme MORAES et al. (1991a), e que o descarte de amostras-problema diminuiu o número necessário para estimar-se a média a um dado nível de probabilidade, mas foi sempre maior que o número necessário para estimar-se qualquer umidade a qualquer tensão, conforme MORAES et al. (1991b).

\section{CONCLUSÕES}

O trabalho mostrou que o conhecimento da variabilidade de propriedades físico-hídricas do solo é condição necessária para estimar a variabilidade de outra propriedade obtida por operações algébricas a partir das primeiras. A utilização do coeficiente de variação mostrou um acréscimo em relação aos dados originais, os coeficientes de simetria e curtose não acompanharam o comportamento daqueles e a sensibilidade dos resultados ficou particularmente alterada com a composição de valores de Capacidade de Campo e ponto de murchamento permanente para obtenção da água disponível, mesmo não se levando em conta a densidade do solo, o que certamente aumentaria a variabilidade observada. Há que se ressaltar que amostrasproblema causaram também influência na água disponível, não sendo responsáveis no entanto, pelo aumento da variabilidade, como ficou evidenciado pelo seu descarte.

Em outras palavras, $o$ ato bastante comum de utilizar-se a caracterização física de um dado solo, mesmo que esta tenha utilizado um mínimo de sete amostras (OLIVEIRA \& MENK, 1984), para obtenção de um terceiro parâmetro de nosso interesse, poderá ser errôneo caso se necessite o mesmo grau de variabilidade ou a mesma sensibilidade de medidas.

O trabalho deixa em aberto a questão da substituição de um número maior de amostras por amostras de maior volume, a fim de se tentar diminuir a variabilidade, talvez com certa economia de trabalho de coleta de amostras, entre outros e que deveria ser motivo de maior atenção pelos pesquisadores. 
TABELA 5. Número necessário de amostras para estimar a média da variável água disponível (AD\% calculada para o ponto de murchamento permanente (PMP) correspondente a $1,5 \times 10^{6}$ e vários valores possíveis de Capacidade de Campo (CC) $\left(1 \times 10^{3} ; 6 \times 10^{3} ; 1 \times 10^{4}\right.$ e $\left.3 \times 10^{4} \mathrm{~Pa}\right)$, com desvio permitido de $10 \%$ e níveis de significância de 90 e $95 \%$.

\begin{tabular}{lllllll}
\hline \hline & \multicolumn{2}{c}{ Níveis de significância } & \multicolumn{2}{c}{ Niveis de significância } & \multicolumn{2}{c}{ Niveis de significância } \\
AD\%(1) & $90 \%$ & $95 \%$ & $90 \%$ & $95 \%$ & $90 \%$ & $95 \%$ \\
\hline AD\% $^{(5)}$ & 15 & 22 & $10^{(9)}$ & $14^{(9)}$ & $8^{(9)}$ & $12^{(9)}$ \\
$\mathrm{AD}^{(9)}$ & $14^{(9)}$ & $20^{(9)}$ & $11^{(9)}$ & $15^{(9)}$ & 9 & 13 \\
$\mathrm{AD} \%^{(7)}$ & 16 & 23 & 12 & 17 & 12 & 17 \\
$\mathrm{AD} \%^{(8)}$ & 35 & 50 & 27 & 38 & $17^{(9)}$ & $24^{(9)}$ \\
\hline \hline
\end{tabular}

(1) $\mathrm{AD} \%=(\mathrm{CC}$ - PMP).100; (2),(3) e (4) 250, 143 e 116 amostras respectivamente; (5), (6), (7) e (8) $1 \times 10^{3}, 6 \times 10^{3}, 1 \times 10^{4}$ e $3 \times 10_{4} \mathrm{~Pa}$ respectivamente e (9) valores de número necessário de amostras mais corretos por satisfazerem ao menos a condição de proximidade da normal.

\section{REFERENCIAS BIBLIOGRAFICAS}

ALJIBURY, F.K. \& EVANS, D.D. Soil sampling for moisture retention and bulk density measurents. Soil Science Society of American Proceeding, Madison, v.25, p.180-183. 1961 .

BORGES, E.A. \& MEDINA, B.F. Correlação entre capacidade de campo determinada in situ e em laboratório. Agropecuária Técnica, Viçosa, v.2, n.1, p.50-61, 1981 .

CASSEL, D.K. \& NIELSEN, D.R. Field capacity and available water capacity. In: KLUTE, A. ed. Methods of Soil Analysis. Part. 1. Physical and minerological methods. Madison, American Society of Agronomy. Soil Science Society of America, 1986. p. 901-926.

CLARK, W.A.V. \& HOSKING, P.L. Statistical methods for geographers. New York. John Wiley \& Sons, 1986. $518 \mathrm{p}$.

CLINE, M.G. Principles of soil sampling. Soil Science, Baltimore, v.58, p.275-288. 1944.

COLMAN, E.A. A laboratory procedure for determining the field capacity of soil. Soil Science, Baltimore, v.67, p.277-283, 1947.

GÓES, L.A.C. Estatistica: uma abordagem decisorial. São Paulo: Saraiva, 1980. 428 p.

GOMES, F.P. 1991. O índice de variação, um substituto vantajoso do coeficiente de variação. Circular Técnica IPEF, Piracicaba, n 178.p.1-4, maio 1991.
HOFFMANN, R. Estatística para economistas. São Paulo: Biblioteca Pioneira de Ciências Sociais, 1980. 379 p.

JAMISON, V.V. \& KROTH, E.M. Available moisture storage capacity, in relation to textural composition and organic matter content of several Missouri soils. Soil Science Society of America Proceedings, Madison, v.22, p.189-192, 1985.

LANDIM, P.M.B. Introduçāo à geoestatística. Rio Claro, UNESP, 1988. 114p. (Publicação Didática 3).

LEAMER, R.W. \& SHAW, B. A simple apparatus for measuring non capillary porosity on an extensive scale. Journal of American Society of Agronomy, Washington, v.33, p.1003-1008, 1941.

MORAES, S.O. Heterogeneidade hidráulica de uma terra roxa estruturada. Piracicaba, 1991, 141 p. Tese (Doutorado) - Escola Superior de Agricultura "Luiz de Queiroz"/Universidade de São Paulo.

MORAES, S.O.; LIBARDI, P.L. \& DOURADO NETO, D. Problemas metodológicos na obtenção da curva de retenção da água no Solo. Scientia Agricola, Piracicaba, v.50, n.3, p.383-392, out./dez., 1993a.

MORAES, S.O.; LIBARDI, P.L., REICHARDT, K. \& BACCHI, O.O.S. Heterogeneidade dos pontos experimentais da curva de retenção da água no solo. Scientia Agricola, Piracicaba, v.50, n.3, p.393-403, out./dez., 1993 b. 
OLIVEIRA, J.B. \& MENK, J.R.F. Latossolos roxos do Estado de São Paulo. Campinas. Instituto Agronômico, 1984. 132 p. (IAC. Boletim Técnico, 82)

PETERSEN, R.G. \& CALVIN, L.D. Sampling. In: BLACK, C.A., ed. Methods of soil analysis. I.Physical and mineralogical methods. Madison, American Society of Agronomy, Soil Science Society of America, 1986. p.33-51.

RATLIFF, L.F.; RITCHIE, J.T. \& CASSEL, D.K. Field-Measured limits of soil water availability as related to laboratory-measured properties. Soil Science Society of America Journal, Madison, v.47, p.770$775,1983$.

REICHARDT, K. Capacidade de campo. Revista Brasileira de Ciência do Solo, Campinas, v.12, p.211-216, 1988 .

RICHARDS, L.A. Methods of measuring soil moisture tension. Soil Science, Madison, v.68, p.85-112, 1949.

SALTER, P.J. \& HAWORTH, F. The available-water capacity of a sandy loam soil. I. A critical comparison of methods of determining the moisture content of soil at field capacity and at permanent wilting percentage. Journal of Soil Science, Oxford, v.12, n.2, p.326$334,1961$.
SILVA, A.P. da. Variabilidade espacial de atributos físicos do solo. Piracicaba, 1988. 98 p. (Doutorado Escola Superior de Agricultura "Luiz de Queiroz"/USP).

SOUZA, L.D. Estimativas e utilização da capacidade de campo. Piracicaba, 1989. 126 p. Dissertação (Mestrado - Escola Superior de Agricultura "Luiz de Queiroz"/Universidade de São Paulo.

STEVENSON, D.S. Unreliabilities of pressure plate 1500 kilopascal data in predicting soil water contents at which plants become wilted in soil-peat mixes: notes. Canadian Journal of Science, Ottawa, v.62, p.415-419, 1982 .

WARRICK, A.W. \& NIELSEN, D.R. Spatial variability of soil physical properties in the field. In: HILLEL, D. ed. Application of soil physics. New York. Academic Press, 1980. 385 p.

Enviado para publicação em 25.01.93

Aceito para publicaçāo em 08.06 .93 